\title{
Delay-Range-Dependent Global Robust Passivity Analysis of Discrete-Time Uncertain Recurrent Neural Networks with Interval Time-Varying Delay
}

\author{
Chien-Yu Lu, ${ }^{1}$ Chin-Wen Liao, ${ }^{1}$ and Hsun-Heng Tsai ${ }^{2}$ \\ ${ }^{1}$ Department of Industrial Education and Technology, National Changhua University of Education, \\ Changhua 500, Taiwan \\ ${ }^{2}$ Department of Biomechatronics Engineering, National Pingtung University of Science E Technology, \\ Pingtung 912, Taiwan
}

Correspondence should be addressed to Chien-Yu Lu, lcy@cc.ncue.edu.tw

Received 11 March 2009; Accepted 25 August 2009

Recommended by Guang Zhang

This paper examines a passivity analysis for a class of discrete-time recurrent neural networks (DRNNs) with norm-bounded time-varying parameter uncertainties and interval time-varying delay. The activation functions are assumed to be globally Lipschitz continuous. Based on an appropriate type of Lyapunov functional, sufficient passivity conditions for the DRNNs are derived in terms of a family of linear matrix inequalities (LMIs). Two numerical examples are given to illustrate the effectiveness and applicability.

Copyright (C) 2009 Chien-Yu Lu et al. This is an open access article distributed under the Creative Commons Attribution License, which permits unrestricted use, distribution, and reproduction in any medium, provided the original work is properly cited.

\section{Introduction}

Recurrent neural networks have been extensively studied in the past decades. Two popular examples are Hopfield neural networks and cellular neural networks. Increasing attention has been draw to the potential applications of recurrent neural networks in information processing systems such as signal processing, model identification, optimization, pattern recognition, and associative memory. However, these successful applications are greatly dependent on the dynamic behavior of recurrent neural networks (RNNs). On the other hand, time delay is inevitably encountered in RNNs, since the interactions between different neurons are asynchronous. Generally, time delays, both constant and time varying, are often encountered in various engineering, biological, and economic systems due to the finite switching speed of amplifiers in electronic networks, or to the finite signal propagation time in biological networks [1]. The existence of time delay could make delayed RNNs be instable or have poor performance. Therefore, many research interests have been attracted to the 
stability analysis for delayed RNNs. A lot of results related to this issue have been reported [2-6].

The theory of passivity plays an important role for analyzing the stability of nonlinear system [7] and has received much attention in the literature from the control community since 1970s [8-14]. It is well known that the passivity theory plays an important role in both electrical network and nonlinear control systems, provides a nice tool for analyzing the stability of system [15], and has found applications in diverse areas such as signal processing, chaos control and synchronization, and fuzzy control. The passivity condition for delayed neural networks with or without time-varying parametric uncertainties using LMIs [16] has been proposed in [17]. By constructing proper Lyapunov functionals and using some analytic techniques, sufficient conditions are given to ensure the passivity of the integro-differential neural networks with time-varying delays in [18]. In [19, 20], the authors studied the delaydependent robust passivity criterion for the delayed cellular neural networks and the delayed recurrent neural networks. It should be pointed out that the aforementioned results are the continuous-time neural networks.

Recently, the stability analysis problems for discrete-time neural networks with time delay have received considerable research interests. For instance in [21], global exponential stability of a class of discrete-time Hopfield neural networks with variable delays is considered. By making use of a difference inequality, a new global exponential stability result is provided. Under different assumptions on the activation functions, a unified linear matrix inequality (LMI) approach has been developed to establish sufficient conditions for the discrete-time recurrent neural networks with interval variable time to be globally exponentially stable in [22]. Delay-dependent results on the global exponential stability problem for discrete-time neural networks with time-varying delays were presented in $[23,24]$, respectively. However, no delay-range-dependent passivity conditions on discretetime uncertain recurrent neural networks with interval time-varying delay are available in the literature and remain essentially open. The objective of this paper is to address this unsolved problem.

The purpose of this paper is to deal with the problem of passivity conditions for discrete-time uncertain recurrent neural networks with interval time-varying delay. The interval time-varying delay includes both lower and upper bounds of delay, and the parameter uncertainties are assumed to be time varying but norm bounded which appear in all the matrices in the state equation. It is then established that the resulting passivity condition can be cast in a linear matrix inequality format which can be conveniently solved by using the numerically effective Matlab LMI Toolbox. In particular, when the interval timedelay factor is known, it is emphasized that delay-range-dependent passivity condition yields more general and practical results. Finally, two numerical examples are given to demonstrate the effectiveness.

Throughout this paper, the notation $X \geq Y(X>Y)$ for symmetric matrices $X$ and $Y$ indicates that the matrix $X-Y$ is positive and semidefinite (resp., positive definite); $Z^{T}$ represents the transpose of matrix $Z$.

\section{Preliminaries}

Consider a discrete-time recurrent neural network with interval time-varying delay described by

$x(k+1)=(A+\Delta A(k)) x(k)+(W+\Delta W(k)) f(x(k))+\left(W_{1}+\Delta W_{1}(k)\right) f(x(k-\tau(k)))+u(k)$, 
where $x(k)=\left(x_{1}(k), x_{2}(k), \ldots, x_{n}(k)\right)^{T}$ is the state vector, $A=\operatorname{diag}\left(a_{1}, a_{2}, \ldots, a_{n}\right)$ with $\left|a_{i}\right|<1, i=1,2, \ldots, n$, is the state feedback coefficient matrix, $W^{n \times n}$ and $W_{1}^{n \times n}$ are the interconnection matrices representing the weighting coefficients of the neurons, $f(x(k))=$ $\left[f_{1}\left(x_{1}(k)\right), \ldots, f_{n}\left(x_{n}(k)\right)\right]^{T} \in R^{n}$ is the neuron activation function with $f(0)=0, \tau(k)$ is the time-varying delay of the system satisfying

$$
\tau_{1} \leq \tau(k) \leq \tau_{2}, \quad k \in \mathrm{N},
$$

where $0 \leq \tau_{1} \leq \tau_{2}$ are known integers. Let $y(k)=f(x(k))$ be the output of the neural networks. $u(k)$ is the input vector. $\Delta A(k), \Delta W(k)$, and $\Delta W_{1}(k)$ are unknown matrices representing time-varying parameter uncertainties, which are assumed to be of the form

$$
\left[\begin{array}{lll}
\Delta A(k) & \Delta W(k) & \Delta W_{1}(k)
\end{array}\right]=\operatorname{MF}(k)\left[\begin{array}{lll}
N_{1} & N_{2} & N_{3}
\end{array}\right],
$$

where $M, N_{1}, N_{2}$, and $N_{3}$ are known real constant matrices, and $F(\cdot): R \rightarrow R^{k \times l}$ is unknown time-varying matrix function satisfying

$$
F^{T}(k) F(k) \leq I, \quad k \in \mathrm{N} .
$$

The uncertain matrices $\Delta A(k), \Delta W(k)$, and $\Delta W_{1}(k)$ are said to be admissible if both $(2.3)$ and (2.4) hold.

In order to obtain our main results, the activation functions in (2.1) are assumed to be bounded and satisfy the following assumption.

Assumption 1. The activation functions $g_{i}(\cdot), i=1,2, \ldots, n$, are globally Lipschitz and monotone nondecreasing; that is, there exist constant scalars $\alpha_{i}$ such that for any $\varsigma_{1}, \varsigma_{2} \in R$ and $\varsigma_{1} \neq \varsigma_{2}$,

$$
0 \leq \frac{g_{i}\left(\varsigma_{1}\right)-g_{i}\left(\varsigma_{2}\right)}{\varsigma_{1}-\varsigma_{2}} \leq \alpha_{i}, \quad i=1,2, \ldots, n
$$

Definition 2.1 ([25]). System (2.1) is called passive if there exists a scalar $\beta \geq 0$ such that

$$
2 \sum_{k=0}^{k_{p}} y^{T}(k) u(k) \geq-\beta \sum_{k=0}^{k_{p}} u^{T}(k) u(k)
$$

for all $k_{p} \geq 0$ and for all solutions of (2.1) with $x(0)=0$.

\section{Mathematical Formulation of the Proposed Approach}

This section explores the globally robust delay-range-dependent passivity conditions of the discrete-time recurrent uncertain neural network with interval time-varying delay given in (2.1). Specially, an LMI approach is employed to solve the robust delay-range-dependent passivity condition if the system in (2.1) is globally asymptotically stable for all admissible 
uncertainties $\Delta A(k), \Delta W(k)$, and $\Delta W_{1}(k)$ satisfying (2.6). The analysis commences by using the LMI approach to develop some results which are essential to introduce the followingLemma 3.1 for the development of our main theorem.

Lemma 3.1. Let $A, D, S, F$, and $P$ be real matrices of appropriate dimensions with $P>0$ and $F$ satisfying $F^{T}(k) F(k) \leq I$. Then the following statements hold.

(a) For any $\varepsilon>0$ and vectors $x, y \in R^{n}$

$$
2 x^{T} D F S y \leq \varepsilon^{-1} x^{T} D D^{T} x+\varepsilon y^{T} S^{T} S y .
$$

(b) For vectors $x, y \in R^{n}$

$$
2 x^{T} D S y \leq x^{T} D P D^{T} x+y^{T} S^{T} P^{-1} S y .
$$

For any matrices $E_{i}, S_{i}, T_{i}$, and $H_{i}(i=1,2, \ldots, 8)$ of appropriate dimensions, it follows from null equations that

$$
\begin{aligned}
& \Phi_{1}=2\left[x^{T}(k) E_{1}+x^{T}(k-\tau(k)) E_{2}+x^{T}\left(k-\tau_{2}\right) E_{3}+x^{T}\left(k-\tau_{1}\right) E_{4}\right. \\
&+\left.f^{T}(x(k)) E_{5}+f^{T}(x(k-\tau(k))) E_{6}+x^{T}(k+1) E_{7}+u^{T}(k) E_{8}\right] \\
& \times {\left[x(k)-x(k-\tau(k))-\sum_{j=k-\tau(k)+1}^{k}(x(j)-x(j-1))\right]=0, } \\
& \Phi_{2}=2\left[x^{T}(k) S_{1}+x^{T}(k-\tau(k)) S_{2}+x^{T}\left(k-\tau_{2}\right) S_{3}+x^{T}\left(k-\tau_{1}\right) S_{4}\right.
\end{aligned}
$$




$$
\begin{aligned}
\Phi_{5}= & 2 f^{T}(x(k)) R_{1} f(x(k))-2 f^{T}(x(k)) R_{1} f(x(k))+2 f^{T}(x(k-\tau(k))) R_{2} x(k-\tau(k)) \\
& -2 f^{T}(x(k-\tau(k))) R_{2} x(k-\tau(k))=0 .
\end{aligned}
$$

To study the globally robust delay-range-dependent passivity conditions of the discrete-time uncertain recurrent neural network with interval time-varying delay, the following theorem reveals that such conditions can be expressed in terms of LMIs.

Theorem 3.2. Under Assumption 1, given scalars $0 \leq \tau_{1}<\tau_{2}$, system (2.1) with interval timevarying delay $\tau(k)$ satisfying $(2.2)$ is globally asymptotically robust stability, if there exist matrices $P>0, Q_{1}>0, Q_{2}>0, Z_{1}>0, Z_{2}>0$, diagonal matrices $R_{1}>0, R_{2}>0$, and matrices $E_{i}, S_{i}, T_{i}$ and $H_{i}(i=1,2, \ldots, 8)$ of appropriate dimensions, a positive scalar $\varepsilon>0$ and a scalar $\beta \geq 0$ such that the following LMI holds:

$$
\left[\begin{array}{cccccc}
\Omega & \tau_{2} E & \tau_{21} S & \tau_{21} T & H M & \varepsilon N \\
\tau_{2} E^{T} & -\tau_{2} Z_{1} & 0 & 0 & 0 & 0 \\
\tau_{21} S^{T} & 0 & -\tau_{21}\left(Z_{1}+Z_{2}\right) & 0 & 0 & 0 \\
\tau_{21} T^{T} & 0 & 0 & -\tau_{21} Z_{2} & 0 & 0 \\
M^{T} H^{T} & 0 & 0 & 0 & -\varepsilon I & 0 \\
\varepsilon N^{T} & 0 & 0 & 0 & 0 & -\varepsilon I
\end{array}\right]<0,
$$

where

$$
\begin{aligned}
& \Omega=\left[\begin{array}{llllllll}
\Omega_{11} & \Omega_{12} & \Omega_{13} & \Omega_{14} & \Omega_{15} & \Omega_{16} & \Omega_{17} & \Omega_{18} \\
\Omega_{12}^{T} & \Omega_{22} & \Omega_{23} & \Omega_{24} & \Omega_{25} & \Omega_{26} & \Omega_{27} & \Omega_{28} \\
\Omega_{13}^{T} & \Omega_{23}^{T} & \Omega_{33} & \Omega_{34} & \Omega_{35} & \Omega_{36} & \Omega_{37} & \Omega_{38} \\
\Omega_{14}^{T} & \Omega_{24}^{T} & \Omega_{34}^{T} & \Omega_{44} & \Omega_{45} & \Omega_{46} & \Omega_{47} & \Omega_{48} \\
\Omega_{15}^{T} & \Omega_{25}^{T} & \Omega_{35}^{T} & \Omega_{45}^{T} & \Omega_{55} & \Omega_{56} & \Omega_{57} & \Omega_{58} \\
\Omega_{16}^{T} & \Omega_{26}^{T} & \Omega_{36}^{T} & \Omega_{46}^{T} & \Omega_{56}^{T} & \Omega_{66} & \Omega_{67} & \Omega_{68} \\
\Omega_{17}^{T} & \Omega_{27}^{T} & \Omega_{37}^{T} & \Omega_{47}^{T} & \Omega_{57}^{T} & \Omega_{67}^{T} & \Omega_{77} & \Omega_{78} \\
\Omega_{18}^{T} & \Omega_{28}^{T} & \Omega_{38}^{T} & \Omega_{48}^{T} & \Omega_{58}^{T} & \Omega_{68}^{T} & \Omega_{78}^{T} & \Omega_{88}
\end{array}\right], \\
& \Omega_{11}=-P+\left(\tau_{21}+1\right) Q_{1}+\tau_{2} Z_{1}+\tau_{21} Z_{2}+E_{1}+E_{1}^{T}+H_{1} A+A^{T} H_{1}^{T}+Q_{2}, \\
& \Omega_{12}=E_{2}^{T}-E_{1}+S_{1}+T_{1}+A^{T} H_{2}^{T}, \quad \Omega_{13}=E_{3}^{T}-S_{1}+A^{T} H_{3}^{T}, \\
& \Omega_{14}=E_{4}^{T}-T_{1}+A^{T} H_{4}^{T}, \quad \Omega_{15}=E_{5}^{T}+H_{1} W_{0}+A^{T} H_{5}^{T}+\Gamma^{T} R_{1}, \\
& \Omega_{16}=E_{6}^{T}+H_{1} W_{1}+A^{T} H_{6}^{T}, \quad \Omega_{17}=E_{7}^{T}-\tau_{2} Z_{1}-\tau_{21} Z_{2}-H_{1}+A^{T} H_{7}^{T}, \\
& \Omega_{18}=E_{8}^{T}+H_{1}+A^{T} H_{8}^{T}, \quad \Omega_{22}=-Q_{1}-E_{2}-E_{2}^{T}+S_{2}+S_{2}^{T}+T_{2}+T_{2}^{T}, \\
& \Omega_{23}=-E_{3}^{T}-S_{2}+S_{3}^{T}+T_{3}^{T}, \quad \Omega_{24}=-E_{4}^{T}-T_{2}+S_{4}^{T}+T_{4}, \\
& \Omega_{25}=-E_{5}^{T}+S_{5}^{T}+T_{5}^{T}+H_{2} W_{0}, \quad \Omega_{26}=-E_{6}^{T}+S_{6}^{T}+T_{6}^{T}+H_{2} W_{1}+R_{2}^{T},
\end{aligned}
$$




$$
\begin{aligned}
& \Omega_{27}=-E_{7}^{T}+S_{7}^{T}+T_{7}^{T}-H_{2}, \quad \Omega_{28}=-E_{8}^{T}+S_{8}^{T}+T_{8}^{T}+H_{2}, \quad \Omega_{33}=-Q_{2}-S_{3}-S_{3}^{T}, \\
& \Omega_{34}=-S_{4}^{T}-T_{3}, \quad \Omega_{35}=-S_{5}^{T}+H_{3} W_{0}, \quad \Omega_{36}=-S_{6}^{T}+H_{3} W_{1}, \quad \Omega_{37}=-S_{7}^{T}-H_{3}, \\
& \Omega_{38}=-S_{8}^{T}+H_{3}, \quad \Omega_{44}=-T_{4}-T_{4}^{T}, \quad \Omega_{45}=T_{5}^{T}+H_{4} W_{0}, \quad \Omega_{46}=-T_{6}^{T}+H_{4} W_{1}, \\
& \Omega_{47}=-T_{7}^{T}-H_{4}, \quad \Omega_{48}=-T_{8}^{T}+H_{4}, \quad \Omega_{55}=-R_{1}-R_{1}^{T}+H_{5} W_{0}+W_{0}^{T} H_{5}^{T}, \\
& \Omega_{56}=H_{5} W_{1}+W_{0}^{T} H_{6}^{T}, \quad \Omega_{57}=-H_{5}+W_{0}^{T} H_{7}^{T}, \quad \Omega_{58}=H_{5}+W_{0}^{T} H_{8}^{T}-I, \\
& \Omega_{66}=-R_{2} \Gamma^{-1}-\left(R_{2} \Gamma^{-1}\right)^{T}+H_{6} W_{1}+W_{1}^{T} H_{6}^{T}, \quad \Omega_{67}=-H_{6}+W_{1}^{T} H_{7}^{T}, \\
& \Omega_{68}=H_{6}+W_{1}^{T} H_{8}^{T}, \quad \Omega_{77}=-H_{7}-H_{7}^{T}+P+\tau_{2} Z_{1}+\tau_{21} Z_{2}, \quad \Omega_{78}=H_{7}-H_{8} ｝ \\
{\Omega_{88}=H_{8}+H_{8}^{T}-\beta I, \quad E=\left[\begin{array}{llllllll}
E_{1}^{T} & E_{2}^{T} & E_{3}^{T} & E_{4}^{T} & E_{5}^{T} & E_{6}^{T} & E_{7}^{T} & E_{8}^{T}
\end{array}\right]^{T},} \\
{S=\left[\begin{array}{llllllll}
S_{1}^{T} & S_{2}^{T} & S_{3}^{T} & S_{4}^{T} & S_{5}^{T} & S_{6}^{T} & S_{7}^{T} & S_{8}^{T}
\end{array}\right]^{T},} \\
{T=\left[\begin{array}{llllllll}
T_{1}^{T} & T_{2}^{T} & T_{3}^{T} & T_{4}^{T} & T_{5}^{T} & T_{6}^{T} & T_{7}^{T} & T_{8}^{T}
\end{array}\right]^{T},} \\
{H=\left[\begin{array}{lllllllll}
H_{1}^{T} & H_{2}^{T} & H_{3}^{T} & H_{4}^{T} & H_{5}^{T} & H_{6}^{T} & H_{7}^{T} & H_{8}^{T}
\end{array}\right]^{T},} \\
{N=\left[\begin{array}{llllllll}
N_{1}^{T} & 0 & 0 & 0 & N_{2}^{T} & N_{3}^{T} & 0 & 0
\end{array}\right]^{T},}
\end{aligned}
$$

in which $\Gamma=\operatorname{diag}\left(\alpha_{1}, \alpha_{2}, \ldots, \alpha_{n}\right), \tau_{21}=\tau_{2}-\tau_{1}$. Then system (2.1) satisfying (3.8) with interval time-varying delay is robust delay-range-dependent passivity condition in the sense of Definition 2.1.

Proof. Choose the Lyapunov-Krasovskii functional candidate for the system in (2.1) as

$$
\begin{aligned}
V(k)= & V_{1}(k)+V_{2}(k)+V_{3}(k)+V_{4}(k) \\
= & x^{T}(k) P x(k)+\sum_{i=-\tau_{2}} \sum_{j=k+i+1}^{k}(x(j)-x(j-1))^{T} Z_{1}(x(j)-x(j-1)) \\
& +\sum_{j=k-\tau(k)}^{k-1} x^{T}(j) Q_{1} x(j)+\sum_{i=-\tau_{2}}^{-1-\tau_{1}} \sum_{j=k+i+1}^{k-1} x^{T}(j) Q_{1} x(j)+\sum_{j=k-\tau_{2}}^{k-1} x^{T}(j) Q_{2} x(j) \\
& +\sum_{i=-\tau_{2}}^{-1-\tau_{1}} \sum_{j=k+i+1}^{k}(x(j)-x(j-1))^{T} Z_{2}(x(j)-x(j-1)) .
\end{aligned}
$$

Then, the difference $\Delta V(k)=V(k+1)-V(k)$ of $V(k)$ along the solution of (2.1) gives

$$
\begin{aligned}
\Delta V_{1}(k)= & x^{T}(k+1) P x(k+1)-x^{T}(k) P x(k), \\
\Delta V_{2}(k)= & \sum_{i=-\tau_{2}}^{-1} \sum_{j=k+i+2}^{k+1}(x(j)-x(j-1))^{T} Z_{1}(x(j)-x(j-1)) \\
& -\sum_{i=-\tau_{2}}^{-1} \sum_{j=k+i+1}^{k}(x(j)-x(j-1))^{T} Z_{1}(x(j)-x(j-1))
\end{aligned}
$$


Discrete Dynamics in Nature and Society

$$
\begin{aligned}
\leq & \tau_{2}(x(k+1)-x(k))^{T} Z_{1}(x(k+1)-x(k)) \\
& -\sum_{j=k-\tau_{2}+1}^{k-\tau(k)}(x(j)-x(j-1))^{T} Z_{1}(x(j)-x(j-1)) \\
& -\sum_{j=k-\tau(k)+1}^{k}(x(j)-x(j-1))^{T} Z_{1}(x(j)-x(j-1)), \\
\Delta V_{3}(k) \leq & x^{T}(k)\left\{Q_{2}+\left[\left(\tau_{2}-\tau_{1}\right)+1\right] Q_{1}\right\} x(k)-x^{T}(k-\tau(k)) Q_{1} x(k-\tau(k)) \\
& -x^{T}\left(k-\tau_{2}\right) Q_{2} x\left(k-\tau_{2}\right), \\
\Delta V_{4}(k)= & \left(\tau_{2}-\tau_{1}\right)(x(k+1)-x(k))^{T} Z_{2}(x(k+1)-x(k)) \\
& -\sum_{j=k-\tau_{2}+1}^{k-\tau(k)}(x(j)-x(j-1))^{T} Z_{2}(x(j)-x(j-1)) \\
& -\sum_{j=k-\tau(k)+1}^{k-\tau_{1}}(x(j)-x(j-1))^{T} Z_{2}(x(j)-x(j-1)) .
\end{aligned}
$$

Defining the following new variables:

$$
\begin{gathered}
\eta(k)=\left[x^{T}(k) x^{T}(k-\tau(k)) x^{T}\left(k-\tau_{2}\right) x^{T}\left(k-\tau_{1}\right) f^{T}(x(k)) f^{T}(x(k-\tau(k))) x^{T}(k+1) u^{T}(k)\right]^{T}, \\
E=\left[\begin{array}{llllllll}
E_{1}^{T} & E_{2}^{T} & E_{3}^{T} & E_{4}^{T} & E_{5}^{T} & E_{6}^{T} & E_{7}^{T} & E_{8}^{T}
\end{array}\right]^{T}, \\
S=\left[\begin{array}{llllllll}
S_{1}^{T} & S_{2}^{T} & S_{3}^{T} & S_{4}^{T} & S_{5}^{T} & S_{6}^{T} & S_{7}^{T} & S_{8}^{T}
\end{array}\right]^{T}, \\
T=\left[\begin{array}{llllllll}
T_{1}^{T} & T_{2}^{T} & T_{3}^{T} & T_{4}^{T} & T_{5}^{T} & T_{6}^{T} & T_{7}^{T} & T_{8}^{T}
\end{array}\right]^{T}, \\
H=\left[\begin{array}{llllllll}
H_{1}^{T} & H_{2}^{T} & H_{3}^{T} & H_{4}^{T} & H_{5}^{T} & H_{6}^{T} & H_{7}^{T} & H_{8}^{T}
\end{array}\right]^{T}, \\
N=\left[\begin{array}{llllllll}
N_{1}^{T} & 0 & 0 & 0 & N_{2}^{T} & N_{3}^{T} & 0 & 0
\end{array}\right]^{T},
\end{gathered}
$$

and combining null equations (3.3)-(3.7), it yields

$$
\begin{aligned}
\Delta V(k) & -2 y^{T}(k) u(k)-\beta u^{T}(k) u(k) \\
= & \Delta V_{1}(k)+\Delta V_{2}(k)+\Delta V_{3}(k)+\Delta V_{4}(k)-2 y^{T}(k) u(k) \\
& -\beta u^{T}(k) u(k)+\Phi_{1}+\Phi_{2}+\Phi_{3}+\Phi_{4}+\Phi_{5} \\
\leq & x^{T}(k+1) P x(k+1)-x^{T}(k) P x(k)+\tau_{2}(x(k+1)-x(k))^{T} Z_{1}(x(k+1)-x(k))
\end{aligned}
$$




$$
\begin{aligned}
& -\sum_{j=k-\tau_{2}+1}^{k-\tau(k)}(x(j)-x(j-1))^{T} Z_{1}(x(j)-x(j-1)) \\
& -\sum_{j=k-\tau(k)+1}^{k}(x(j)-x(j-1))^{T} Z_{1}(x(j)-x(j-1)) \\
& +x^{T}(k)\left\{Q_{2}+\left[\left(\tau_{2}-\tau_{1}\right)+1\right] Q_{1}\right\} x(k)-x^{T}(k-\tau(k)) Q_{1} x(k-\tau(k)) \\
& -x^{T}\left(k-\tau_{2}\right) Q_{2} x\left(k-\tau_{2}\right)+\left(\tau_{2}-\tau_{1}\right)(x(k+1)-x(k))^{T} Z_{2}(x(k+1)-x(k)) \\
& -\sum_{j=k-\tau_{2}+1}^{k-\tau(k)}(x(j)-x(j-1))^{T} Z_{2}(x(j)-x(j-1)) \\
& -\sum_{j=k-\tau(k)+1}^{k-\tau_{1}}(x(j)-x(j-1))^{T} Z_{2}(x(j)-x(j-1)) \\
& +2 \eta^{T}(k) E\left[x(k)-x(k-\tau(k))-\sum_{j=k-\tau(k)+1}^{k}(x(j)-x(j-1))\right] \\
& +2 \eta^{T}(k) S\left[x(k-\tau(k))-x\left(k-\tau_{2}\right)-\sum_{j=k-\tau_{2}+1}^{k-\tau(k)}(x(j)-x(j-1))\right] \\
& -2 \eta^{T}(k) T\left[x\left(k-\tau_{1}\right)-x(k-\tau(k))-\sum_{j=k-\tau(k)+1}^{k-\tau_{1}}(x(j)-x(j-1))\right] \\
& -2 \eta^{T}(k) H[x(k+1)-(A+\Delta A(k)) x(k)-(W+\Delta W(k)) f(x(k)) \\
& \left.-\left(W_{1}+\Delta W_{1}(k)\right) f(x(k-\tau(k)))-u(k)\right] \\
& +2 f^{T}(x(k)) R_{1} f(x(k))-2 f^{T}(x(k)) R_{1} f(x(k))+2 f^{T}(x(k-\tau(k))) R_{2} x(k-\tau(k)) \\
& -2 f^{T}(x(k-\tau(k))) R_{2} x(k-\tau(k))-2 y^{T}(k) u(k)-\beta u^{T}(k) u(k) .
\end{aligned}
$$

Moreover,

$$
\begin{aligned}
& -2 \eta^{T}(k) E \sum_{j=k-\tau(k)+1}^{k}(x(j)-x(j-1)) \\
& \quad \leq \tau_{2} \eta^{T}(k) E Z_{1}^{-1} E^{T} \eta(k)+\sum_{j=k-\tau(k)+1}^{k}(x(j)-x(j-1))^{T} Z_{1}(x(j)-x(j-1)), \\
& -2 \eta^{T}(k) S \sum_{j=k-\tau_{2}+1}^{k-\tau(k)}(x(j)-x(j-1)) \\
& \quad \leq\left(\tau_{2}-\tau_{1}\right) \eta^{T}(k) S\left(Z_{1}+Z_{2}\right)^{-1} S^{T} \eta(k)+\sum_{j=k-\tau_{2}+1}^{k-\tau(k)}(x(j)-x(j-1))^{T}\left(Z_{1}+Z_{2}\right)(x(j)-x(j-1)),
\end{aligned}
$$




$$
\begin{aligned}
& 2 \eta^{T}(k) T \sum_{j=k-\tau(k)+1}^{k-\tau_{1}}(x(j)-x(j-1)) \\
& \quad \leq\left(\tau_{2}-\tau_{1}\right) \eta^{T}(k) T Z_{2}^{-1} T^{T} \eta(k)+\sum_{j=k-\tau(k)+1}^{k-\tau_{1}}(x(j)-x(j-1))^{T} Z_{2}(x(j)-x(j-1)) .
\end{aligned}
$$

Using Assumption 1 and noting that $R_{1}>0$ and $R_{2}>0$ are diagonal matrices, one has

$$
\begin{gathered}
2 f^{T}(x(k)) R_{1} f(x(k)) \leq 2 f^{T}(x(k)) R_{1} \Gamma x(k), \\
-2 f^{T}(x(k-\tau(k))) R_{2} x(k-\tau(k)) \leq-2 f^{T}(x(k-\tau(k))) R_{2} \Gamma^{-1} f(x(k-\tau(k))),
\end{gathered}
$$

where $\Gamma=\operatorname{diag}\left(\alpha_{1}, \alpha_{2}, \ldots, \alpha_{n}\right)$.

Following from Lemma 3.1(a) results in

$$
\begin{aligned}
& 2 \eta^{T}(k) H\left(\Delta A(k) x(k)+\Delta W_{0}(k) f(x(k))+\Delta W_{1}(k) f(x(k-\tau(k)))\right) \\
& \quad=2 \eta^{T}(k) H M F(k) N^{T} \eta(k) \leq \varepsilon^{-1} \eta^{T}(k) H M M^{T} H^{T} \eta(k)+\varepsilon \eta^{T}(k) N N^{T} \eta(k) .
\end{aligned}
$$

Substituting (3.14)-(3.19) into (3.13), it is not difficult to deduce that

$$
\begin{aligned}
& \Delta V(k)-2 y^{T}(k) u(k)-\beta u^{T}(k) u(k) \\
& \quad \leq \eta^{T}(k)\left[\Omega+\tau_{2} E Z_{1}^{-1} E^{T}+\tau_{21} S\left(Z_{1}+Z_{2}\right)^{-1} S^{T}+\tau_{21} T Z_{2}^{-1} T^{T}+\varepsilon^{-1} H M M^{T} H^{T}+\varepsilon N N^{T}\right] \eta(k) .
\end{aligned}
$$

Using the Schur complement to the (3.20) and in view of LMI (3.8), it follows that

$$
\Delta V(k)-2 y^{T}(k) u(k)-\beta u^{T}(k) u(k)<0 .
$$

It follows from (3.21) that

$$
2 \sum_{k=0}^{k_{p}} y^{T}(k) u(k) \geq V\left(x\left(k_{p}\right)\right)-V(x(0))-\beta \sum_{k=0}^{k_{p}} u^{T}(k) u(k),
$$

for $x(0)=0$, one has $V(x(0))=0$, so (2.6) holds, and hence the system is robust delayrange-dependent passivity condition in the sense of Definition 2.1. This completes the proof of Theorem 3.2.

Remark 3.3. Theorem 3.2 provides a sufficient passivity condition for the globally robust stability of the discrete-time uncertain recurrent neural network with interval time-varying delay given in (2.1) and proposes a delay-range-dependent criterion. Even for $\tau_{1}=0$, the result in Theorem 3.2 may lead to the delay-dependent stability criteria. In fact, if $Z_{2}=\varepsilon_{1} I$, 
with $\varepsilon_{1}>0$, being sufficiently small scalar, $T_{i}=0, i=1,2, \ldots, 8, E_{4}=0, S_{4}=0, H_{4}=0$, Theorem 3.2 yields the following delay-dependent passivity criterion.

Corollary 3.4. Under Assumption 1, given scalars $\tau_{2}>0, \tau_{1}=0$, system (2.1) with time-varying delay satisfying (2.3) is globally asymptotically robust stability, if there exist matrices $P>0, Q_{1}>0$, $Q_{2}>0, Z_{1}>0$, diagonal matrices $R_{1}>0, R_{2}>0$, and matrices $E_{i}, S_{i}$, and $H_{i}(i=1,2, \ldots, 8)$ of appropriate dimensions and $E_{4}=S_{4}=H_{4}=0$, a positive scalar $\varepsilon>0$, and a scalar $\beta \geq 0$ such that the following LMI holds:

$$
\left[\begin{array}{ccccc}
\Omega & \tau_{2} E & \tau_{2} S & H M & \varepsilon N \\
\tau_{2} E^{T} & -\tau_{2} Z_{1} & 0 & 0 & 0 \\
\tau_{2} S^{T} & 0 & -\tau_{2} Z_{1} & 0 & 0 \\
M^{T} H^{T} & 0 & 0 & -\varepsilon I & 0 \\
\varepsilon N^{T} & 0 & 0 & 0 & -\varepsilon I
\end{array}\right]<0
$$

where

$$
\begin{aligned}
& \Omega=\left[\begin{array}{lllllll}
\Omega_{11} & \Omega_{12} & \Omega_{13} & \Omega_{15} & \Omega_{16} & \Omega_{17} & \Omega_{18} \\
\Omega_{12}^{T} & \Omega_{22} & \Omega_{23} & \Omega_{25} & \Omega_{26} & \Omega_{27} & \Omega_{28} \\
\Omega_{13}^{T} & \Omega_{23}^{T} & \Omega_{33} & \Omega_{35} & \Omega_{36} & \Omega_{37} & \Omega_{38} \\
\Omega_{15}^{T} & \Omega_{25}^{T} & \Omega_{35}^{T} & \Omega_{55} & \Omega_{56} & \Omega_{57} & \Omega_{58} \\
\Omega_{16}^{T} & \Omega_{26}^{T} & \Omega_{36}^{T} & \Omega_{56}^{T} & \Omega_{66} & \Omega_{67} & \Omega_{68} \\
\Omega_{17}^{T} & \Omega_{27}^{T} & \Omega_{37}^{T} & \Omega_{57}^{T} & \Omega_{67}^{T} & \Omega_{77} & \Omega_{78} \\
\Omega_{18}^{T} & \Omega_{28}^{T} & \Omega_{38}^{T} & \Omega_{58}^{T} & \Omega_{68}^{T} & \Omega_{78}^{T} & \Omega_{88}
\end{array}\right], \\
& \Omega_{11}=-P+\left(\tau_{2}+1\right) Q_{1}+\tau_{2} Z_{1}+E_{1}+E_{1}^{T}+H_{1} A+A^{T} H_{1}^{T}+Q_{2}, \\
& \Omega_{12}=E_{2}^{T}-E_{1}+S_{1}+A^{T} H_{2}^{T}, \quad \Omega_{13}=E_{3}^{T}-S_{1}+A^{T} H_{3}^{T}, \\
& \Omega_{15}=E_{5}^{T}+H_{1} W_{0}+A^{T} H_{5}^{T}+\Gamma^{T} R_{1}, \quad \Omega_{16}=E_{6}^{T}+H_{1} W_{1}+A^{T} H_{6}^{T}, \\
& \Omega_{17}=E_{7}^{T}-\tau_{2} Z_{1}-H_{1}+A^{T} H_{7}^{T}, \quad \Omega_{18}=E_{8}^{T}+H_{1}+A^{T} H_{8}^{T}, \\
& \Omega_{22}=-Q_{1}-E_{2}-E_{2}^{T}+S_{2}+S_{2}^{T}, \quad \Omega_{23}=-E_{3}^{T}-S_{2}+S_{3}^{T}, \quad \Omega_{25}=-E_{5}^{T}+S_{5}^{T}+H_{2} W_{0}, \\
& \Omega_{26}=-E_{6}^{T}+S_{6}^{T}+H_{2} W_{1}+R_{2}^{T}, \quad \Omega_{27}=-E_{7}^{T}+S_{7}^{T}-H_{2}, \quad \Omega_{28}=-E_{8}^{T}+S_{8}^{T}+H_{2}, \\
& \Omega_{33}=-Q_{2}-S_{3}-S_{3}^{T}, \quad \Omega_{35}=-S_{5}^{T}+H_{3} W_{0}, \quad \Omega_{36}=-S_{6}^{T}+H_{3} W_{1}, \Omega_{37}=-S_{7}^{T}-H_{3}, \\
& \Omega_{38}=-S_{8}^{T}+H_{3}, \quad \Omega_{55}=-R_{1}-R_{1}^{T}+H_{5} W_{0}+W_{0}^{T} H_{5}^{T}, \quad \Omega_{56}=H_{5} W_{1}+W_{0}^{T} H_{6}^{T}, \\
& \Omega_{57}=-H_{5}+W_{0}^{T} H_{7}^{T}, \quad \Omega_{58}=H_{5}+W_{0}^{T} H_{8}^{T}-I, \\
& \Omega_{66}=-R_{2} \Gamma^{-1}-\left(R_{2} \Gamma^{-1}\right)^{T}+H_{6} W_{1}+W_{1}^{T} H_{6}^{T}, \quad \Omega_{67}=-H_{6}+W_{1}^{T} H_{7}^{T},
\end{aligned}
$$




$$
\begin{aligned}
& \Omega_{68}=H_{6}+W_{1}^{T} H_{8}^{T}, \quad \Omega_{77}=-H_{7}-H_{7}^{T}+P+\tau_{2} Z_{1}, \quad \Omega_{78}=H_{7}-H_{8}, \\
& \Omega_{88}=H_{8}+H_{8}^{T}-\beta I, \quad E=\left[\begin{array}{llllllllll}
E_{1}^{T} & E_{2}^{T} & E_{3}^{T} & E_{5}^{T} & E_{6}^{T} & E_{7}^{T} & E_{8}^{T}
\end{array}\right]^{T}, \\
& S=\left[\begin{array}{lllllllllll}
S_{1}^{T} & S_{2}^{T} & S_{3}^{T} & S_{5}^{T} & S_{6}^{T} & S_{7}^{T} & S_{8}^{T}
\end{array}\right]^{T}, \quad H=\left[\begin{array}{lllllllll}
H_{1}^{T} & H_{2}^{T} & H_{3}^{T} & H_{5}^{T} & H_{6}^{T} & H_{7}^{T} & H_{8}^{T}
\end{array}\right]^{T}, \\
& N=\left[\begin{array}{lllllll}
N_{1}^{T} & 0 & 0 & N_{2}^{T} & N_{3}^{T} & 0 & 0
\end{array}\right]^{T} .
\end{aligned}
$$

Therefore, the discrete-time recurrent neural network with time-varying delay in (2.1) (i.e., the lower bounds $\tau_{1}=0$ and the given upper bounds $\tau_{2}$ ) approaches globally robustly delay-dependent passivity condition in the sense of Definition 2.1.

Remark 3.5. In the stochastic context, robust delay-dependent passivity conditions are studied in [26] for discrete-time stochastic neural networks with time-varying delays. In this paper, however, robust delay-range-dependent passivity conditions are studied in the deterministic context. It should be noted that deterministic systems and stochastic systems have different properties and need to be dealt with separately. The results given in Theorem 3.2 provide an LMI approach to the robust delay-range-dependent passivity conditions for deterministic discrete-time recurrent neural networks with interval time-varying delay, which is new and represents a contribution to recurrent neural networks systems.

Two numerical examples are now presented to demonstrate the usefulness of the proposed approach.

\section{Examples}

Example 4.1. Consider the following discrete-time uncertain recurrent neural network:

$x(k+1)=(A+\Delta A(k)) x(k)+(W+\Delta W(k)) f(x(k))+\left(W_{1}+\Delta W_{1}(k)\right) f(x(k-\tau(k)))+u(k)$,

where

$$
\begin{gathered}
A=\left[\begin{array}{cc}
0.2 & 0 \\
0 & 0.14
\end{array}\right], \quad W=\left[\begin{array}{cc}
0.2 & 0.25 \\
0.25 & 0.1
\end{array}\right], \quad W_{1}=\left[\begin{array}{cc}
-0.2 & 0.1 \\
0.1 & 0.3
\end{array}\right], \\
M=\left[\begin{array}{ll}
0.2 & 0.1 \\
0.1 & 0.2
\end{array}\right], \quad N_{1}=\left[\begin{array}{ll}
0.1 & 0.2 \\
0.1 & 0.2
\end{array}\right], \quad N_{2}=\left[\begin{array}{cc}
0.3 & 0.1 \\
0 & 0.1
\end{array}\right], \quad N_{3}=\left[\begin{array}{ll}
0.2 & 0.1 \\
0.1 & 0.1
\end{array}\right] .
\end{gathered}
$$

The activation functions in this example are assumed to satisfy Assumption 1 with $\alpha_{1}=$ $0.5633, \alpha_{2}=0.0478$. For interval time-varying delay, the best approached values of $\beta$ by Theorem 3.2, for the given upper bound $\tau_{2}=10$ and the various lower bounds $\tau_{1}$, are listed inTable 1 by the Matlab LMI Control Toolbox. Therefore, using Theorem 3.2, the discrete-time uncertain recurrent neural network with interval time-varying delay (4.1) satisfies robustly delay-range-dependent passivity conditions in the sense of Definition 2.1 for various $\beta$ levels. 
Example 4.2. Consider the discrete-time uncertain recurrent neural network with the following parameters:

$$
\begin{array}{ccc}
A & =\left[\begin{array}{ccc}
0.1 & 0 & 0 \\
0 & 0.1 & 0 \\
0 & 0 & 0.2
\end{array}\right], & W=\left[\begin{array}{ccc}
0.1 & -0.1 & 0.2 \\
0.1 & -0.2 & 0.3 \\
-0.1 & 0 & -0.1
\end{array}\right], \quad W_{1}=\left[\begin{array}{ccc}
-0.1 & 0.2 & 0.1 \\
-0.1 & 0.4 & 0.2 \\
0.2 & -0.1 & 0.4
\end{array}\right], \\
M & =\left[\begin{array}{ccc}
0.1 & 0 & 0.1 \\
0.1 & 0.3 & 0 \\
0.2 & 0.1 & 0
\end{array}\right], \quad N_{1}=\left[\begin{array}{ccc}
0 & 0.02 & 0.02 \\
-0.1 & 0.1 & 0.1 \\
-0.1 & 0.1 & 0.1
\end{array}\right], \quad N_{2}=\left[\begin{array}{ccc}
0 & -0.02 & 0.02 \\
0.1 & -0.1 & 0 \\
0.1 & 0 & -0.1
\end{array}\right], \\
N_{3} & =\left[\begin{array}{ccc}
0.01 & 0.02 & 0 \\
-0.1 & 0.3 & 0.1 \\
0.1 & 0 & 0.2
\end{array}\right] .
\end{array}
$$

The activation functions in this example are assumed to satisfy Assumption 1 with $\alpha_{1}=0.034$, $\alpha_{2}=0.429, \alpha_{3}=0.508$. By the Matlab LMI Control Toolbox, it can be verified that Corollary 3.4 in this paper is feasible solution for all delays $0 \leq \tau(k) \leq 15$ (i.e., the lower bound $\tau_{1}=0$ and the upper bound $\tau_{2}=15$ ) as follows:

$$
\begin{aligned}
& P=\left[\begin{array}{ccc}
2.3189 & -0.2657 & -0.0023 \\
-0.2657 & 2.2981 & -0.0574 \\
-0.0023 & -0.0574 & 2.3612
\end{array}\right], \quad Q_{1}=\left[\begin{array}{ccc}
0.0565 & -0.0056 & 0.0005 \\
-0.0056 & 0.0679 & -0.0052 \\
0.0005 & -0.0052 & 0.0657
\end{array}\right], \\
& Z_{1}=\left[\begin{array}{ccc}
0.0199 & -0.0038 & 0.0008 \\
-0.0038 & 0.0150 & 0.0002 \\
0.0008 & 0.0002 & 0.0144
\end{array}\right], \quad Z_{2}=\left[\begin{array}{ccc}
0.0215 & -0.0042 & 0.0009 \\
-0.0042 & 0.0158 & 0.0002 \\
0.0009 & 0.0002 & 0.0153
\end{array}\right], \\
& R_{1}=\left[\begin{array}{ccc}
1.8998 & 0 & 0 \\
0 & 1.6298 & 0 \\
0 & 0 & 1.8378
\end{array}\right], \quad R_{2}=\left[\begin{array}{ccc}
1.2991 & 0 & 0 \\
0 & 0.2980 & 0 \\
0 & 0 & 0.2635
\end{array}\right], \\
& \varepsilon=3.4160, \quad \beta=5.0169 \text {. }
\end{aligned}
$$

Thus, by Corollary 3.4, the discrete-time uncertain recurrent neural network with timevarying delay in (2.1) (i.e., the lower bound $\tau_{1}=0$ and the given upper bound $\tau_{2}=15$ ) attains globally robustly delay-dependent passivity condition in the sense of Definition 2.1. 
Table 1: The various lower bounds $\tau_{1}$ and $\beta$ levels for the given upper bound $\tau_{2}=10$.

\begin{tabular}{lc}
\hline$\tau_{1}$ & $\beta$ \\
\hline 0 & 5.9189 \\
1 & 5,9228 \\
2 & 5.8145 \\
3 & 5.9832 \\
4 & 5.8014 \\
5 & 5.9522 \\
6 & 5.9744 \\
7 & 6.0126 \\
8 & 6.0186 \\
9 & 6.1851 \\
\hline
\end{tabular}

\section{Conclusions}

This study has investigated the problem of globally robust passivity conditions for a discretetime recurrent uncertain neural network with interval time-varying delay. A sufficient condition for the solvability of this problem, which takes into account the range for the time delay, has been established that the passivity conditions can be cast in linear matrix inequalities format. It has been shown that the bound for the time-varying delay in a range which ensures that the discrete-time recurrent uncertain neural network with interval timevarying delay attains globally robust passivity conditions can be obtained by solving a convex optimization problem. Two numerical examples have been presented to demonstrate the effectiveness of the proposed approach.

\section{References}

[1] S. Arik, "Global asymptotic stability of a larger class of neural networks with constant time delay," Physics Letters A, vol. 311, no. 6, pp. 504-511, 2003.

[2] I. Gyóri and F. Hartung, "Stability analysis of a single neuron model with delay," Journal of Computational and Applied Mathematics, vol. 157, no. 1, pp. 73-92, 2003.

[3] E. Kaszkurewicz and A. Bhaya, "On a class of globally stable neural circuits," IEEE Transactions on Circuits and Systems I, vol. 41, no. 2, pp. 171-174, 1994.

[4] J. Cao and J. Wang, "Global asymptotic stability of a general class of recurrent neural networks with time-varying delays," IEEE Transactions on Circuits and Systems I, vol. 50, no. 1, pp. 34-44, 2003.

[5] M. Joy, "Results concerning the absolute stability of delayed neural networks," Neural Networks, vol. 13, no. 6, pp. 613-616, 2000.

[6] S. Xu, Y. Chu, and J. Lu, "New results on global exponential stability of recurrent neural networks with time-varying delays," Physics Letters A, vol. 352, no. 4-5, pp. 371-379, 2006.

[7] L. O. Chua, "Passivity and complexity," IEEE Transactions on Circuits and Systems I, vol. 46, no. 1, pp. 71-82, 1999.

[8] L. Xie, M. Fu, and H. Li, "Passivity analysis for uncertain signal processing systems," in Proceedings of the IEEE International Conference on Signal Processing, vol. 46, pp. 2394-2403, 1998.

[9] M. S. Mahmoud and A. Ismail, "Passivity and passification of time-delay systems," Journal of Mathematical Analysis and Applications, vol. 292, no. 1, pp. 247-258, 2004.

[10] E. Fridman and U. Shaked, "On delay-dependent passivity," IEEE Transactions on Automatic Control, vol. 47, no. 4, pp. 664-669, 2002.

[11] T. Hayakawa, W. M. Haddad, J. M. Bailey, and N. Hovakimyan, "Passivity-based neural network adaptive output feedback control for nonlinear nonnegative dynamical systems," IEEE Transactions on Neural Networks, vol. 16, no. 2, pp. 387-398, 2005.

[12] S.-I. Niculescu and R. Lozano, "On the passivity of linear delay systems," IEEE Transactions on Automatic Control, vol. 46, no. 3, pp. 460-464, 2001. 
[13] J. C. Travieso-Torres, M. A. Duarte-Mermoud, and J. L. Estrada, "Tracking control of cascade systems based on passivity: the non-adaptive and adaptive cases," ISA Transactions, vol. 45, no. 3, pp. 435-445, 2006.

[14] L. Keviczky and Cs. Bányász, "Robust stability and performance of time-delay control systems," ISA Transactions, vol. 46, no. 2, pp. 233-237, 2007.

[15] R. Lozano, B. Brogliato, O. Egeland, and B. Maschke, Dissipative Systems Analysis and Control: Theory and Application, Communications and Control Engineering Series, Springer, London, UK, 2000.

[16] S. Boyd, L. El Ghaoui, E. Feron, and V. Balakrishnan, Linear Matrix Inequalities in System and Control Theory, vol. 15 of SIAM Studies in Applied Mathematics, SIAM, Philadelphia, Pa, USA, 1994.

[17] C. Li and X. Liao, "Passivity analysis of neural networks with time delay," IEEE Transactions on Circuits and Systems II, vol. 52, no. 8, pp. 471-475, 2005.

[18] X. Lou and B. Cui, "Passivity analysis of integro-differential neural networks with time-varying delays," Neurocomputing, vol. 70, no. 4-6, pp. 1071-1078, 2007.

[19] J. H. Park, "Further results on passivity analysis of delayed cellular neural networks," Chaos, Solitons $\mathcal{E}$ Fractals, vol. 34, no. 5, pp. 1546-1551, 2007.

[20] Q. Song and Z. Wang, "New results on passivity analysis of uncertain neural networks with timevarying delays," International Journal of Computer Mathematics. In press.

[21] Q. Zhang, X. Wei, and J. Xu, "On global exponential stability of discrete-time Hopfield neural networks with variable delays," Discrete Dynamics in Nature and Society, vol. 2007, Article ID 67675, 9 pages, 2007.

[22] Y. Liu, Z. Wang, A. Serrano, and X. Liu, "Discrete-time recurrent neural networks with time-varying delays: exponential stability analysis," Physics Letters A, vol. 362, no. 5-6, pp. 480-488, 2007.

[23] W.-H. Chen, X. Lu, and D.-Y. Liang, "Global exponential stability for discrete-time neural networks with variable delays," Physics Letters A, vol. 358, no. 3, pp. 186-198, 2006.

[24] J. Liang, J. Cao, and D. W. C. Ho, "Discrete-time bidirectional associative memory neural networks with variable delays," Physics Letters A, vol. 335, no. 2-3, pp. 226-234, 2005.

[25] M. S. Mahmoud, Robust Control and Filtering for Time-Delay Systems, vol. 5 of Control Engineering, Marcel Dekker, New York, NY, USA, 2000.

[26] Q. Song, J. Liang, and Z. Wang, "Passivity analysis of discrete-time stochastic neural networks with time-varying delays," Neurocomputing, vol. 72, no. 7-9, pp. 1782-1788, 2009. 


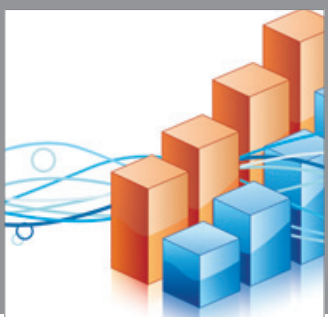

Advances in

Operations Research

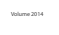

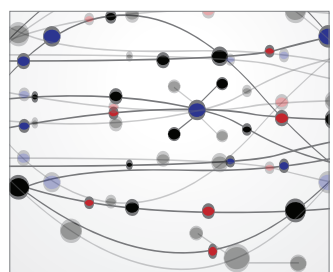

\section{The Scientific} World Journal
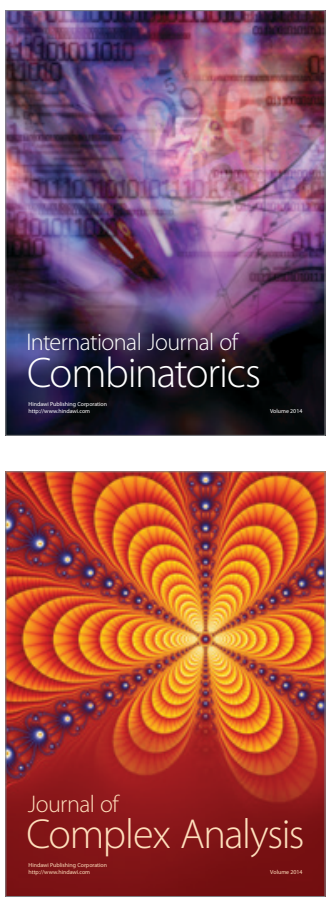

International Journal of

Mathematics and

Mathematical

Sciences
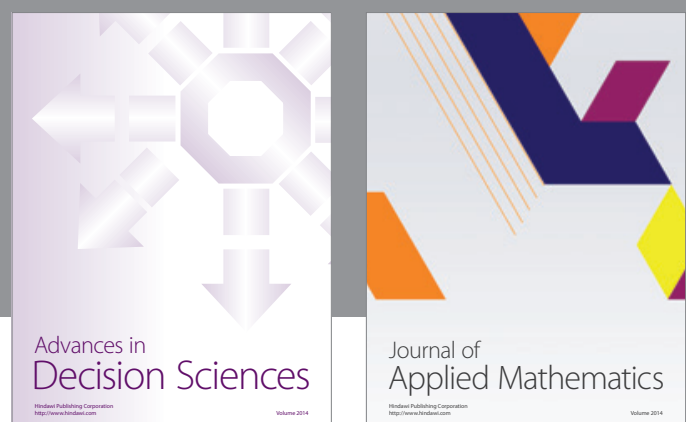

Journal of

Applied Mathematics
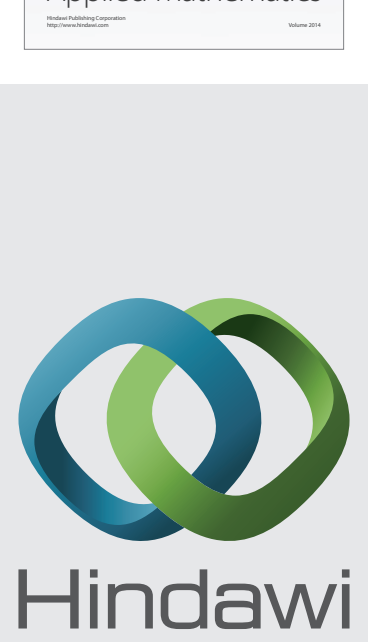

Submit your manuscripts at http://www.hindawi.com
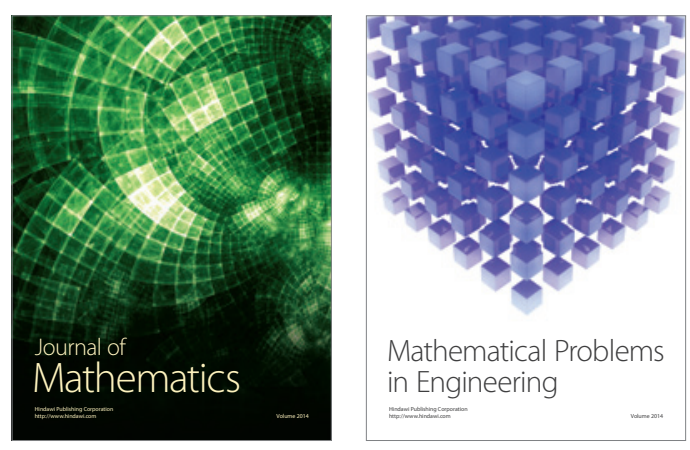

Mathematical Problems in Engineering
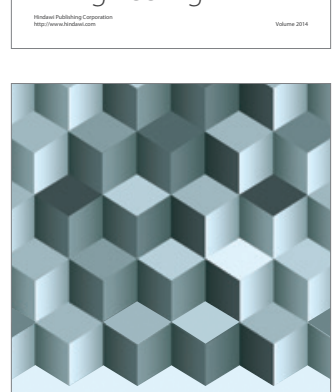

Journal of

Function Spaces
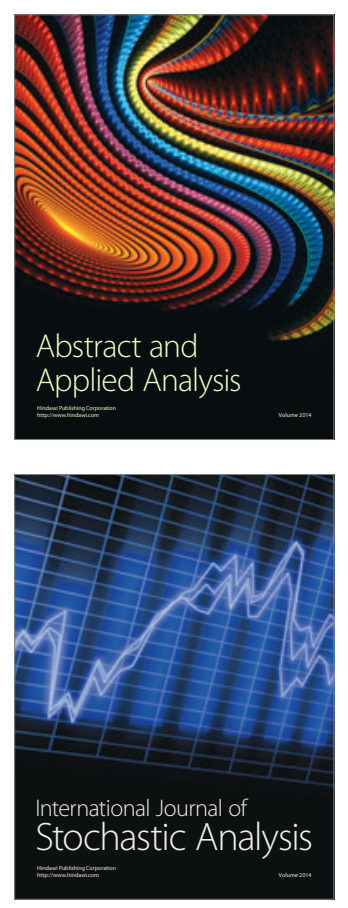

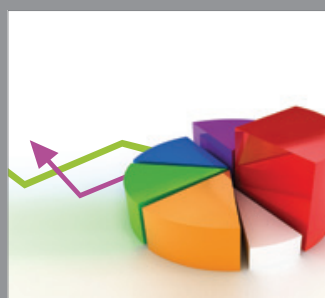

ournal of

Probability and Statistics

Promensencen
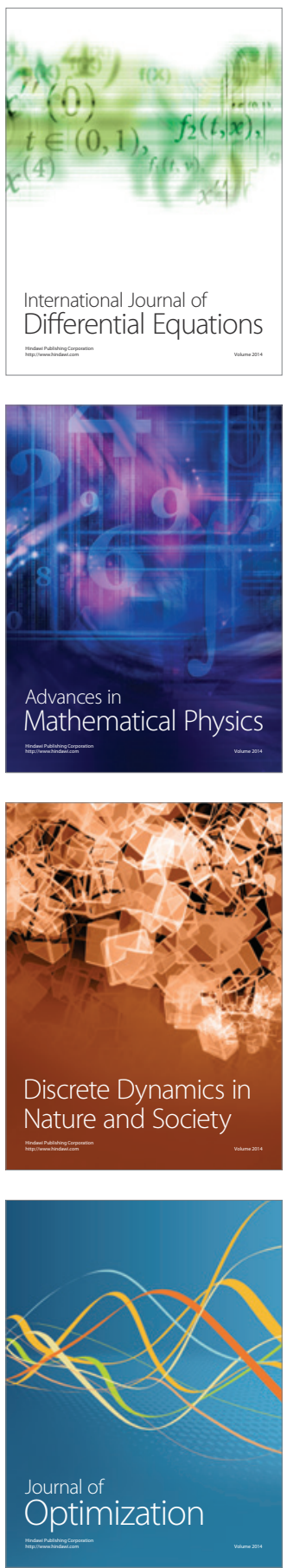